\title{
El aprendizaje basado en tareas en la producción oral del idioma francés (Nivel
} B1)

\author{
Task-based learning in the oral production of the French language (Level B1) \\ Aprendizagem baseada em tarefas na produção oral da língua francesa (nível B1)
}

\author{
Gabriela M. Moso-Mena ${ }^{\mathrm{I}}$ \\ gmmoso@uce.edu.ec \\ Amilcar O. González-Díaz II \\ aogonzalez@uce.edu.ec \\ Myriam M. Hurtado-Angamarca III \\ moryhurt@hotmail.com \\ Edison S. Sanguña-Loachamin ${ }^{\text {IV }}$ \\ essanguna@uce.edu.ec
}

Recibido: 30 de enero de 2017 * Corregido: 20 de febrero de 2017 * Aceptado: 20 junio de 2017

I. Magister en Educación Superior y Equidad de Género; Licenciada en Ciencias de la Educación Mención Plurilingüe; Universidad Central del Ecuador, Quito, Ecuador.

II. Magister en Lingüística y Didáctica de la Enseñanza de Idiomas Extranjeros; Diploma Superior de la Enseñanza de Inglés como Segunda Lengua; Licenciado en Ciencias de la Educación Mención Ingles; Universidad Central del Ecuador, Quito, Ecuador.

III. Magister en Lingüística y Didáctica de la Enseñanza de Idiomas Extranjeros; Licenciada en Ciencias de la Educación Mención Ingles; Universidad Central del Ecuador, Quito, Ecuador.

IV. Magister en Docencia Universitaria y Administración Educativa; Especialista en Diseño Curricular por Competencias; Licenciado en Ciencias de la Educación Mención: Plurilingüe; Universidad Central del Ecuador, Quito, Ecuador. 


\section{Resumen}

La presente investigación tiene como objetivo analizar la influencia del aprendizaje basado en tareas en el desarrollo de la producción oral del idioma francés nivel B1. Se plantearon la hipótesis alterna y la hipótesis nula. Se realizó una profunda investigación teórica de las dos variables. La metodología se enfocó hacia la investigación cuanti-cualitativa y el nivel planteado se orientó a la investigación explicativa cuyo nivel se basó en la investigación cuasi experimental estableciendo el grupo de experimental y control a los que se aplicó dos instrumentos el pre test y el post test. Los datos obtenidos, sirvieron para obtener el cálculo de la media aritmética y la desviación típica. Posteriormente se hizo la prueba $\mathrm{Z}$ y se determinó la aceptación de la hipótesis alterna. Para la variable independiente se aplicó la técnica de la entrevista al área de francés de la Carrera Plurilingüe cuyos resultados corroboraron al aporte significativo de método del aprendizaje basado en tareas en la producción oral de los/las estudiantes del quinto semestre.

Palabras clave: Influencia del aprendizaje; pre test; post test; francés. 


\section{Abstract}

The present research aims to analyze the influence of task based learning on the development of French language oral production B1 level. The alternative hypothesis and the null hypothesis were proposed. A thorough theoretical research of the two variables was carried out. The methodology was focused on quantitative-qualitative research and the level was oriented to the explanatory research whose level was based on quasi-experimental research establishing the experimental and control group to which two instruments were applied; the pre test and the post test. The obtained data served to obtain the media arithmetic calculation and the standard deviation. Subsequently the $\mathrm{Z}$ test was performed and the acceptance of the alternate hypothesis was determined. For the independent variable, the interview technique was applied to the French area of the Plurilingual Career whose results corroborated the significant contribution of the task-based learning method in the oral production of fifth semester students.

Key words: Influence of learning; pre test; post test; French. 


\section{Introducción.}

La presente investigación, se desarrolla bajo un contexto educativo a nivel de pregrado en donde la formación en el idioma francés, es parte fundamental dentro del perfil de egreso de los/las estudiantes de la Carrera Plurilingüe de la Universidad Central de Ecuador. Como es de conocimiento público, el Ministerio de Educación ha firmado una carta de entendimiento con la Embajada de Francia para incluir el idioma francés dentro de la malla curricular de Bachillerato General Unificado (BGU). Asimismo, el Ministerio de Educación del Ecuador aprobó un proyecto piloto para la enseñanza del francés en tres colegios del país (Quito, Guayaquil y Loja) y se emitió un Acuerdo Ministerial para establecer el idioma francés como segunda lengua extranjera. Por otro lado, existe un convenio de cooperación lingüística entre la Universidad Central del Ecuador y la Embajada de Francia y para que los/las estudiantes de la Carrera Plurilingüe puedan participar en este programa, deben tener poseer competencias lingüísticas equivalentes a partir del nivel B1 según el Marco Común Europeo de Referencia para la Lenguas.

Esta investigación se enfoca en los y las estudiantes de quinto semestre de la Carrera Plurilingüe, ya que los mismos, necesitan profundizar sus conocimientos en el idioma francés priorizando la producción oral, siendo la competencia lingüística que más dificultades enfrentan los y las estudiantes.

La Carrera Plurilingüe de la Universidad Central del Ecuador, forma profesionales de idiomas en inglés y francés; y viendo la necesidad de que los/las futuros docentes necesitan estar en constante capacitación sobre metodologías innovadoras, este trabajo de investigación estuvo direccionado en el método aprendizaje basado en tareas "L’apprentissage par tâches” para el 
desarrollo de la producción oral del idioma francés (Nivel B1). Este método, aportó de manera significativa al progreso en la producción oral de los y las estudiantes sujetos de estudio.

Este método engloba todos los elementos para aprender eficazmente un idioma extranjero. Toma en cuenta los objetivos del idioma, se orienta a lo que los y las estudiantes necesitan aprender y qué actividades comunicativas son de utilidad para lograr la comunicación oral.

La investigación es de tipo cuasi experimental y se utilizaron dos grupos (experimental y control) a quienes se les aplicó el pre-test y post-test y se calculó la correlación a través del puntaje Z. Se obtuvo como producto final una propuesta metodológica basada en el aprendizaje por tareas.

El contenido de este trabajo se lo realizó según el esquema que presenta el Instituto de Investigación y Posgrado de la Facultad de Filosofía, Letras y Ciencias de la Educación.

El objetivo de la presente investigación es analizar la Influencia del Aprendizaje Basado en Tareas "L’apprentissage par tâches" en el desarrollo de la Producción Oral del Idioma Francés (Nivel B1) en los y las estudiantes del Quinto Semestre de la Carrera Plurilingüe de la Facultad de Filosofía, Letras y Ciencias de la Educación de la Universidad Central del Ecuador en el semestre 2017 - 2017. 


\section{Materiales y métodos.}

\section{Enfoque de la investigación.}

La presente investigación se enfoca bajo el modelo mixto. (Sampieri Hernández, Collado Fernández, \& Lucio Baptista, 2003) definen el enfoque mixto como un conjunto de procesos sistemáticos, empíricos y críticos de investigación que involucran la recolección y el análisis de datos cuantitativos y cualitativos, así como su integración y discusión conjunta, para realizar deducciones del producto de toda la información obtenida y lograr un mayor entendimiento del fenómeno de estudio.

La mezcla de los dos enfoques potencia el desarrollo del conocimiento, la construcción de teorías y la resolución de problemas. Ambos son empíricos porque recogen datos del fenómeno que estudian. En la presente investigación el modelo dominante es el enfoque cuantitativo el cual conserva componentes del enfoque cualitativo.

El enfoque cuantitativo usa la recolección de datos para probar hipótesis con base en la medición numérica y el análisis estadístico para establecer esquemas de comportamiento. Ofrece la posibilidad de generalizar los resultados más amplios, otorga control sobre los fenómenos y un punto de vista de conteo de magnitudes de éstos.

Según (Lavayen, 2007) esta investigación genera una medida. Se enfoca en el estudio y análisis de la realidad por medio de procesos relacionados con la medición. Se puede manipular las variables y así obtener explicaciones diferenciadas según las hipótesis planteadas. Por lo antes expuesto, se aplicó el método del aprendizaje basado en tareas para influir sobre la variable 
dependiente, la producción oral nivel B1. Los resultados de esta investigación se basan en la estadística obtenida mediante la aplicación de pre test y post test.

El enfoque cualitativo utiliza recolección de datos sin medición numérica para descubrir o afinar preguntas de investigación y puede o no probar hipótesis en su proceso de interpretación. Da profundidad a los datos, la contextualización del ambiente o entorno, los detalles y las experiencias únicas. También aporta un punto de vista “natural” de los fenómenos, así como flexibilidad.

La muestra es una unidad de análisis o un grupo de personas, contextos, eventos, sucesos, comunidades, etc., sobre la cual se recolectan los datos sin que necesariamente sean representativos del universo o población que se estudia. Se habla de características y cualidades de los indicadores las que se reflejan en la entrevista al cuerpo docente de francés, los mismos que expresaron su punto de vista sobre preguntas relacionadas a la variable independiente, el aprendizaje basado en tareas.

\section{Modalidad del Trabajo de Grado}

Para el cumplimiento de los objetivos diseñados en la presente investigación, se trabajó bajo la siguiente modalidad:

1. Revisión bibliográfica y elaboración del marco teórico.

2. Planteamiento y formulación del problema.

3. Elaboración de la matriz de operacionalización de variables.

4. Formulación de objetivos general y específicos.

5. Formulación de la hipótesis alterna y nula. 
6. Caracterización de la población y selección de los grupos de estudio (experimental y de control).

7. Selección de las técnicas de investigación.

8. Selección y elaboración de los instrumentos de la investigación.

9. Trabajo de campo.

10. Codificación y procesamiento de la información.

11. Análisis de datos.

12. Elaboración de conclusiones y recomendaciones.

13. Formulación de la propuesta.

\section{Nivel de la Investigación}

Según el nivel, es una investigación explicativa que busca encontrar las causas que provocan ciertos fenómenos y cuáles son sus consecuencias. Se lleva a cabo cuando el/la investigador/a se fija objetivos para estudiar el porqué de las cosas. Se analizan la causa-efecto de la relación entre variables por medio de la prueba de las hipótesis. En este caso se busca la relación entre la variable independiente, aprendizaje basado en tareas y la variable dependiente, producción oral nivel B1.

El estudio correlacional tiene como finalidad medir el grado de relación que existe entre dos o más variables, dicho de otro modo, cómo una variable explica la otra variable. El objetivo de este estudio es saber cómo se desenvuelve una variable al conocer el comportamiento de otras variables relacionadas a ella. 
Según (Tamayo y Tamayo, 2003) se refiere al “grado de relación (no causal) que existe entre dos o más variables. Para realizar este tipo de estudio, primero se debe medir las variables y luego, mediante pruebas de hipótesis correlacionales acompañadas de la aplicación de técnicas estadísticas, se estima la correlación". La investigación correlacional: es aquel tipo de estudio que persigue medir el grado de relación existente entre dos o más conceptos o variables.

\section{Tipo de Investigación}

El tipo de la presente investigación es cuasi experimental el mismo que es particularmente útil para estudiar problemas en los cuales no se puede tener control absoluto de las situaciones, pero se pretende tener el mayor control posible, aun cuando se estén usando grupos ya formados.

Se utiliza dos grupos: el grupo experimental y el grupo de control. A los dos grupos se aplicó un pre test en base a la variable dependiente (Producción Oral del francés). Se estableció el índice de correlación a través del puntaje $\mathrm{Z}$ y se aplicó al grupo experimental el método del aprendizaje basado en tareas (variable independiente) para comprobar si la misma incide sobre la producción oral (variable dependiente). La investigación cuasi experimental se utiliza cuando no es posible asignar al azar los sujetos de los grupos de investigación que reciben un tratamiento experimental.

También es una investigación de campo para comprender y resolver alguna situación, necesidad o problema en un contexto determinado. El/la investigador/a trabaja en el ambiente natural en que conviven las personas y las fuentes consultadas, de las que obtienen los datos más relevantes a ser analizados, son individuos, grupos y representaciones de las organizaciones científicas no experimentales dirigidas a descubrir relaciones e interacciones entre variables sociológicas, psicológicas y educativas en estructuras sociales reales y cotidianas. 
Es una investigación bibliográfica porque se buscó información sobre investigaciones ya existentes para apoyar el tema de investigación. Las fuentes consultadas estuvieron relacionadas con los objetos de investigación y se establecieron bajo un criterio de exhaustividad ya que se indagaron todas las fuentes posibles y suficientes para fundamentar la investigación. De igual manera, las mismas fueron lo suficientemente actuales para evidenciar los últimos avances del tema de estudio también sus antecedentes más pertinentes en referencia a hechos sucedidos en el pasado y en el presente.

\section{Población}

(Herrera, Medina, Naranjo, \& Proaño, 2002) Mencionan que la presente investigación es no probabilística y cuya muestra es decisional la misma que toma en cuenta el criterio del/ de la investigador/a ya que es quien decide justificadamente quienes forman parte de la muestra. Se trabajó con los siguientes sujetos de estudio. Considerando los grupos de control (18) y experimental (31) suman en total 49 sujetos de estudio y fue el mismo tamaño de la población y de la muestra. Por el tipo de investigación no se calculó la muestra. 


\begin{tabular}{||c||c||}
\hline \multicolumn{1}{|c||}{\begin{tabular}{c||} 
ESTUDIANTES DE LA CARRERA PLURILINGÜE \\
SEMESTRE 2017 -2017
\end{tabular}} & NÚMERO \\
QUINTO SEMESTRE A & 31 \\
\hline \hline QUINTO SEMESTRE B & 18 \\
\hline \hline TOTAL DE ESTUDIANTES & $\mathbf{4 9}$ \\
\hline \hline DOCENTES TITULARES DE FRANCÉS DE LA CARRERA \\
PLURILINGÜE
\end{tabular}

Tabla $N^{\circ} 1 .-$ Estudiantes y Docentes de la Carrera Plurilingüe

\section{Resultados.}

Análisis e Interpretación del Pre Test del Grupo Experimental y Grupo de Control

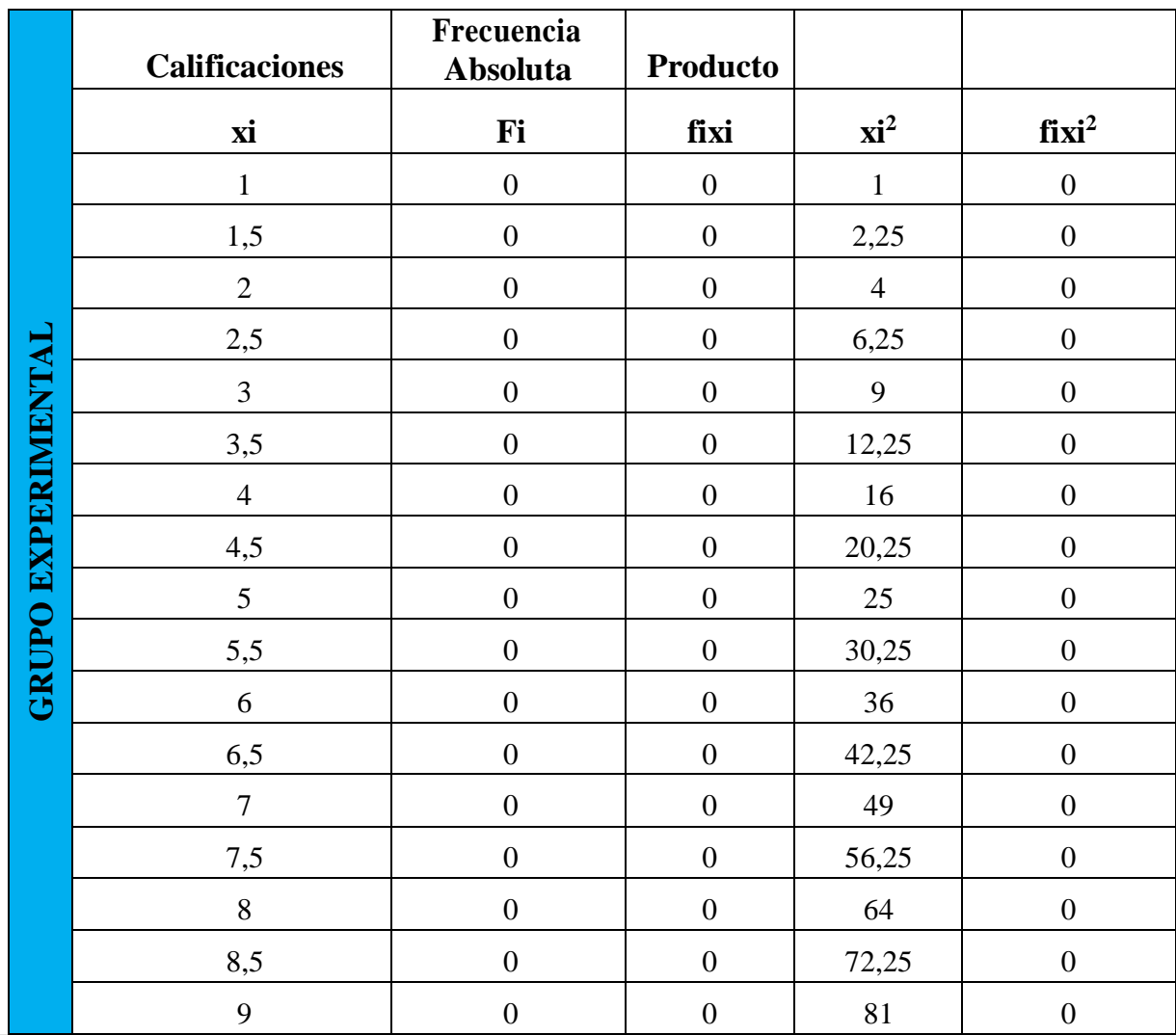


Dom. Cien., ISSN: 2477-8818

Vol. 3, núm. 4, octubre, 2017, pp. 402-425

El aprendizaje basado en tareas en la producción oral del idioma francés (Nivel B1)

\begin{tabular}{|c|c|c|c|c|}
\hline 9,5 & 0 & 0 & 90,25 & 0 \\
\hline 10 & 0 & 0 & 100 & 0 \\
\hline 10,5 & 0 & 0 & 110,25 & 0 \\
\hline 11 & 0 & 0 & 121 & 0 \\
\hline 11,5 & 0 & 0 & 132,25 & 0 \\
\hline 12 & 1 & 12 & 144 & 144 \\
\hline 12,5 & 0 & 0 & 156,25 & 0 \\
\hline 13 & 1 & 13 & 169 & 169 \\
\hline 13,5 & 1 & 13,5 & 182,25 & 182,25 \\
\hline 14 & 2 & 28 & 196 & 392 \\
\hline 14,5 & 3 & 43,5 & 210,25 & 630,75 \\
\hline 15 & 1 & 15 & 225 & 225 \\
\hline 15,5 & 2 & 31 & 240,25 & 480,5 \\
\hline 16 & 1 & 16 & 256 & 256 \\
\hline 16,5 & 3 & 49,5 & 272,25 & 816,75 \\
\hline 17 & 3 & 51 & 289 & 867 \\
\hline 17,5 & 1 & 17,5 & 306,25 & 306,25 \\
\hline 18 & 4 & 72 & 324 & 1296 \\
\hline 18,5 & 2 & 37 & 342,25 & 684,5 \\
\hline 19 & 2 & 38 & 361 & 722 \\
\hline 19,5 & 2 & 39 & 380,25 & 760,5 \\
\hline 20 & 2 & 40 & 400 & 800 \\
\hline 20,5 & 0 & 0 & 420,25 & 0 \\
\hline 21 & 0 & 0 & 441 & 0 \\
\hline 21,5 & 0 & 0 & 462,25 & 0 \\
\hline 22 & 0 & 0 & 484 & 0 \\
\hline 22,5 & 0 & 0 & 506,25 & 0 \\
\hline 23 & 0 & 0 & 529 & 0 \\
\hline 23,5 & 0 & 0 & 552,25 & 0 \\
\hline 24 & 0 & 0 & 576 & 0 \\
\hline 24,5 & 0 & 0 & 600,25 & 0 \\
\hline \multirow[t]{2}{*}{25} & 0 & 0 & 625 & 0 \\
\hline & $\Sigma \mathbf{f i}=31$ & $\mathbf{\Sigma}$ fixi $=516$ & & $\boldsymbol{\Sigma} \mathbf{f i x i}^{2}=8732,5$ \\
\hline
\end{tabular}

Cuadro $N^{\circ}$ 2.- Registro General de Calificaciones del Pre Test Grupo Experimental 
Dom. Cien., ISSN: 2477-8818

Vol. 3, núm. 4, octubre, 2017, pp. 402-425

El aprendizaje basado en tareas en la producción oral del idioma francés (Nivel B1)

\begin{tabular}{|c|c|c|c|c|}
\hline Calificaciones & Frecuencia Absoluta & Producto & & \\
\hline xi & $\mathbf{F i}$ & fixi & $\mathbf{x i}^{2}$ & fixi $^{2}$ \\
\hline 1 & 0 & 0 & 1 & 0 \\
\hline 1,5 & 0 & 0 & 2,25 & 0 \\
\hline 2 & 0 & 0 & 4 & 0 \\
\hline 2,5 & 0 & 0 & 6,25 & 0 \\
\hline 3 & 0 & 0 & 9 & 0 \\
\hline 3,5 & 0 & 0 & 12,25 & 0 \\
\hline 4 & 0 & 0 & 16 & 0 \\
\hline 4,5 & 0 & 0 & 20,25 & 0 \\
\hline 5 & 0 & 0 & 25 & 0 \\
\hline 5,5 & 0 & 0 & 30,25 & 0 \\
\hline 6 & 0 & 0 & 36 & 0 \\
\hline 6,5 & 0 & 0 & 42,25 & 0 \\
\hline 7 & 0 & 0 & 49 & 0 \\
\hline 7,5 & 0 & 0 & 56,25 & 0 \\
\hline 8 & 0 & 0 & 64 & 0 \\
\hline 8,5 & 0 & 0 & 72,25 & 0 \\
\hline 9 & 0 & 0 & 81 & 0 \\
\hline 9,5 & 0 & 0 & 90,25 & 0 \\
\hline 10 & 1 & 10 & 100 & 100 \\
\hline 10,5 & 0 & 0 & 110,25 & 0 \\
\hline 11 & 0 & 0 & 121 & 0 \\
\hline 11,5 & 0 & 0 & 132,25 & 0 \\
\hline 12 & 1 & 12 & 144 & 144 \\
\hline 12,5 & 0 & 0 & 156,25 & 0 \\
\hline 13 & 0 & 0 & 169 & 0 \\
\hline 13,5 & 0 & 0 & 182,25 & 0 \\
\hline 14 & 1 & 14 & 196 & 196 \\
\hline 14,5 & 2 & 29 & 210,25 & 420,5 \\
\hline 15 & 1 & 15 & 225 & 225 \\
\hline 15,5 & 1 & 15,5 & 240,25 & 240,25 \\
\hline 16 & 3 & 48 & 256 & 768 \\
\hline 16,5 & 2 & 33 & 272,25 & 544,5 \\
\hline 17 & 1 & 17 & 289 & 289 \\
\hline 17,5 & 2 & 35 & 306,25 & 612,5 \\
\hline 18 & 1 & 18 & 324 & 324 \\
\hline 18,5 & 0 & 0 & 342,25 & 0 \\
\hline 19 & 1 & 19 & 361 & 361 \\
\hline 19,5 & 1 & 19,5 & 380,25 & 380,25 \\
\hline
\end{tabular}




\begin{tabular}{|c|c|c|c|c|}
\hline 20 & 0 & 0 & 400 & 0 \\
\hline 20,5 & 0 & 0 & 420,25 & 0 \\
\hline 21 & 0 & 0 & 441 & 0 \\
\hline 21,5 & 0 & 0 & 462,25 & 0 \\
\hline 22 & 0 & 0 & 484 & 0 \\
\hline 22,5 & 0 & 0 & 506,25 & 0 \\
\hline 23 & 0 & 0 & 529 & 0 \\
\hline 23,5 & 0 & 0 & 552,25 & 0 \\
\hline 24 & 0 & 0 & 576 & 0 \\
\hline 24,5 & 0 & 0 & 600,25 & 0 \\
\hline 25 & 0 & 0 & 625 & 0 \\
\hline & $\mathbf{\Sigma f i}=18$ & $\boldsymbol{\Sigma}$ fixi $=285$ & & $\boldsymbol{\Sigma}$ fixi $^{2}=4605$ \\
\hline
\end{tabular}

\section{Cuadro $N^{\circ}$ 2.- Registro General de Calificaciones del Pre Test Grupo Control}

\section{$\sigma:$ Desviación típica.}

\section{$\Sigma$ f: Sumatoria de las frecuencias.}

\section{N: Número total de casos.}

\section{$\Sigma x$ : variables (calificaciones)}

\section{n: número total de datos.}

Los resultados del Pre test en el grupo experimental y grupo de control revelan que no existe gran diferencia entre los grupos ya que la mayoría del grupo experimental obtuvo una media aritmética de 16,65/25 mientras que el grupo de control obtuvo 15,83/25.

\section{Análisis e Interpretación del Post Test del Grupo Experimental y Grupo de Control}

\begin{tabular}{|c|c|c|c|c|c|}
\hline \multirow{7}{*}{ 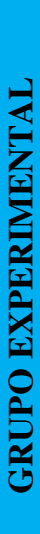 } & $\begin{array}{c}\text { Calificacione } \\
\text { s }\end{array}$ & $\begin{array}{c}\text { Frecuencia } \\
\text { Absoluta }\end{array}$ & Producto & & \\
\hline & $\mathbf{x i}$ & Fi & fixi & $\mathbf{x i}^{2}$ & $f_{i x i^{2}}$ \\
\hline & 1 & 0 & 0 & 1 & 0 \\
\hline & 1,5 & 0 & 0 & 2,25 & 0 \\
\hline & 2 & 0 & 0 & 4 & 0 \\
\hline & 2,5 & 0 & 0 & 6,25 & 0 \\
\hline & 3 & 0 & 0 & 9 & 0 \\
\hline
\end{tabular}


Dom. Cien., ISSN: 2477-8818

Vol. 3, núm. 4, octubre, 2017, pp. 402-425

El aprendizaje basado en tareas en la producción oral del idioma francés (Nivel B1)

\begin{tabular}{|c|c|c|c|c|}
\hline 3,5 & 0 & 0 & 12,25 & 0 \\
\hline 4 & 0 & 0 & 16 & 0 \\
\hline 4,5 & 0 & 0 & 20,25 & 0 \\
\hline 5 & 0 & 0 & 25 & 0 \\
\hline 5,5 & 0 & 0 & 30,25 & 0 \\
\hline 6 & 0 & 0 & 36 & 0 \\
\hline 6,5 & 0 & 0 & 42,25 & 0 \\
\hline 7 & 0 & 0 & 49 & 0 \\
\hline 7,5 & 0 & 0 & 56,25 & 0 \\
\hline 8 & 0 & 0 & 64 & 0 \\
\hline 8,5 & 0 & 0 & 72,25 & 0 \\
\hline 9 & 0 & 0 & 81 & 0 \\
\hline 9,5 & 0 & 0 & 90,25 & 0 \\
\hline 10 & 0 & 0 & 100 & 0 \\
\hline 10,5 & 0 & 0 & 110,25 & 0 \\
\hline 11 & 0 & 0 & 121 & 0 \\
\hline 11,5 & 0 & 0 & 132,25 & 0 \\
\hline 12 & 1 & 12 & 144 & 144 \\
\hline 12,5 & 0 & 0 & 156,25 & 0 \\
\hline 13 & 1 & 13 & 169 & 169 \\
\hline 13,5 & 1 & 13,5 & 182,25 & 182,25 \\
\hline 14 & 2 & 28 & 196 & 392 \\
\hline 14,5 & 3 & 43,5 & 210,25 & 630,75 \\
\hline 15 & 1 & 15 & 225 & 225 \\
\hline 15,5 & 2 & 31 & 240,25 & 480,5 \\
\hline 16 & 1 & 16 & 256 & 256 \\
\hline 16,5 & 3 & 49,5 & 272,25 & 816,75 \\
\hline 17 & 3 & 51 & 289 & 867 \\
\hline 17,5 & 1 & 17,5 & 306,25 & 306,25 \\
\hline
\end{tabular}


Dom. Cien., ISSN: 2477-8818

Vol. 3, núm. 4, octubre, 2017, pp. 402-425

El aprendizaje basado en tareas en la producción oral del idioma francés (Nivel B1)

\begin{tabular}{|c|c|c|c|c|}
\hline 18 & 4 & 72 & 324 & 1296 \\
\hline 18,5 & 2 & 37 & 342,25 & 684,5 \\
\hline 19 & 2 & 38 & 361 & 722 \\
\hline 19,5 & 2 & 39 & 380,25 & 760,5 \\
\hline 20 & 2 & 40 & 400 & 800 \\
\hline 20,5 & 0 & 0 & 420,25 & 0 \\
\hline 21 & 0 & 0 & 441 & 0 \\
\hline 21,5 & 0 & 0 & 462,25 & 0 \\
\hline 22 & 0 & 0 & 484 & 0 \\
\hline 22,5 & 0 & 0 & 506,25 & 0 \\
\hline 23 & 0 & 0 & 529 & 0 \\
\hline 23,5 & 0 & 0 & 552,25 & 0 \\
\hline 24 & 0 & 0 & 576 & 0 \\
\hline 24,5 & 0 & 0 & 600,25 & 0 \\
\hline \multirow[t]{2}{*}{25} & 0 & 0 & 625 & 0 \\
\hline & $\Sigma \mathbf{f i}=31$ & $\mathbf{\Sigma f i x i}=516$ & & $\Sigma \mathbf{f i x i}^{2}=8732,5$ \\
\hline
\end{tabular}

\section{Cuadro $N^{\circ}$ 3.- Registro General de Calificaciones del Pre Test Grupo Experimental}

\begin{tabular}{|c|c|c|c|c|}
\hline Calificaciones & Frecuencia Absoluta & Producto & & \\
\hline $\mathbf{x i}$ & $\mathbf{F i}$ & fixi & $\mathbf{x i}^{2}$ & fixi $^{2}$ \\
\hline 1 & 0 & 0 & 1 & 0 \\
\hline 1,5 & 0 & 0 & 2,25 & 0 \\
\hline 隶 & 0 & 0 & 4 & 0 \\
\hline 2,5 & 0 & 0 & 6,25 & 0 \\
\hline ? & 0 & 0 & 9 & 0 \\
\hline 3,5 & 0 & 0 & 12,25 & 0 \\
\hline 4 & 0 & 0 & 16 & 0 \\
\hline 4,5 & 0 & 0 & 20,25 & 0 \\
\hline 5 & 0 & 0 & 25 & 0 \\
\hline
\end{tabular}


Dom. Cien., ISSN: 2477-8818

Vol. 3, núm. 4, octubre, 2017, pp. 402-425

El aprendizaje basado en tareas en la producción oral del idioma francés (Nivel B1)

\begin{tabular}{|c|c|c|c|c|}
\hline 5,5 & 0 & 0 & 30,25 & 0 \\
\hline 6 & 0 & 0 & 36 & 0 \\
\hline 6,5 & 0 & 0 & 42,25 & 0 \\
\hline 7 & 0 & 0 & 49 & 0 \\
\hline 7,5 & 0 & 0 & 56,25 & 0 \\
\hline 8 & 0 & 0 & 64 & 0 \\
\hline 8,5 & 0 & 0 & 72,25 & 0 \\
\hline 9 & 0 & 0 & 81 & 0 \\
\hline 9,5 & 0 & 0 & 90,25 & 0 \\
\hline 10 & 1 & 10 & 100 & 100 \\
\hline 10,5 & 0 & 0 & 110,25 & 0 \\
\hline 11 & 0 & 0 & 121 & 0 \\
\hline 11,5 & 0 & 0 & 132,25 & 0 \\
\hline 12 & 1 & 12 & 144 & 144 \\
\hline 12,5 & 0 & 0 & 156,25 & 0 \\
\hline 13 & 0 & 0 & 169 & 0 \\
\hline 13,5 & 0 & 0 & 182,25 & 0 \\
\hline 14 & 1 & 14 & 196 & 196 \\
\hline 14,5 & 2 & 29 & 210,25 & 420,5 \\
\hline 15 & 1 & 15 & 225 & 225 \\
\hline 15,5 & 1 & 15,5 & 240,25 & 240,25 \\
\hline 16 & 3 & 48 & 256 & 768 \\
\hline 16,5 & 2 & 33 & 272,25 & 544,5 \\
\hline 17 & 1 & 17 & 289 & 289 \\
\hline 17,5 & 2 & 35 & 306,25 & 612,5 \\
\hline 18 & 1 & 18 & 324 & 324 \\
\hline 18,5 & 0 & 0 & 342,25 & 0 \\
\hline 19 & 1 & 19 & 361 & 361 \\
\hline 19,5 & 1 & 19,5 & 380,25 & 380,25 \\
\hline 20 & 0 & 0 & 400 & 0 \\
\hline
\end{tabular}




\begin{tabular}{|c|c|c|c|c|}
\hline 20,5 & 0 & 0 & 420,25 & 0 \\
\hline 21 & 0 & 0 & 441 & 0 \\
\hline 21,5 & 0 & 0 & 462,25 & 0 \\
\hline 22 & 0 & 0 & 484 & 0 \\
\hline 22,5 & 0 & 0 & 506,25 & 0 \\
\hline 23 & 0 & 0 & 529 & 0 \\
\hline 23,5 & 0 & 0 & 552,25 & 0 \\
\hline 24 & 0 & 0 & 576 & 0 \\
\hline 24,5 & 0 & 0 & 600,25 & 0 \\
\hline 25 & 0 & $\mathbf{2 f i x i}=285$ & & $\mathbf{\Sigma}$ \\
\hline
\end{tabular}

\section{Cuadro $N^{\circ}$ 4.- Registro General de Calificaciones del Pre Test Grupo Control}

Los resultados del Post test en el grupo experimental $(21,03)$ evidencian una diferencia significativa en comparación a grupo de control (16,81). En consecuencia, se evidencia claramente la influencia de la hipótesis alterna en el cálculo de la media aritmética.

\begin{tabular}{|c||l|c||c||}
\hline No & \multicolumn{1}{|c|}{ Evaluaciones } & Media aritmética & Desviación estándar( $\boldsymbol{\sigma})$ \\
\hline \hline 1 & Pre Test & 16,65 & 2,11 \\
\hline \hline 2 & Post Test & 21,03 & 2,49 \\
\hline \hline & PROMEDIO GENERAL & $\mathbf{1 8 , 8 4}$ & $\mathbf{2 , 3 0}$ \\
\hline
\end{tabular}

\section{Cuadro $N^{\circ}$ 5.- Registro de Notas Grupo Experimental}

Se compara la media aritmética y la desviación del grupo experimental en donde se puede observar que la media aritmética del post test es mayor que la media aritmética del pre test. También se evidencia que la desviación típica del pre test es menor, esto quiere decir que, los datos son más concentrados alrededor la su media aritmética. 


\begin{tabular}{|c|l||c|c||}
\hline No & \multicolumn{1}{|c|}{ Evaluaciones } & Media aritmética & Desviación estándar( $\boldsymbol{\sigma})$ \\
\hline \hline 1 & Pre Test & 15,83 & 2,29 \\
\hline \hline 2 & Post Test & 16,81 & 2,15 \\
\hline & PROMEDIO GENERAL & $\mathbf{1 6 , 3 2}$ & $\mathbf{2 , 2 2}$ \\
\hline
\end{tabular}

\section{Cuadro $N^{\circ}$ 6.- Registro de Notas Grupo Control}

Se compara la media aritmética y desviación típica del grupo de control, se observa que la media aritmética del post test es mayor que la media aritmética del pre test pero esta media aritmética no tiene mucha diferencia entre estos dos valores a comparación del grupo experimental. Asimismo, se evidencian los valores de la desviación estándar del pre test y post test al comparar estos valores se observa que la desviación estándar del post test está más concentrada en la media aritmética.

El tipo de estadística que se utilizó fue el puntaje Z. Se realizó el registro de calificaciones del Pre Test y Post Test tanto del grupo experimental como del grupo de control y para el análisis estadístico se tomó en cuenta: la desviación típica, la sumatoria de las frecuencias, el número total de casos, las variables (calificaciones) y el número total de datos. Igualmente se ejecutó el cálculo de la media aritmética del grupo experimental y control.

Luego de este procedimiento se procesó el análisis y prueba de las hipótesis tanto en lenguaje usual como en lenguaje matemático. En el registro de notas del grupo experimental y control se calculó la media aritmética y la desviación estándar del pre test y post test obtenido como resultado el promedio general de los dos grupos. Asimismo, se determinó los 
valores críticos y zonas de rechazo a través del cálculo de la prueba paramétrica $\mathrm{Z}$ y se presentó las dos fórmulas que rechazan la hipótesis nula evidenciando el 95\% de confiabilidad.

Se realizó el cálculo de la prueba paramétrica $\mathrm{Z}$ y finalmente se tomó la decisión estadística al comparar el valor $\mathrm{Z}$ calculado y el valor de $\mathrm{Z}$ teórico se determinó que la hipótesis nula está en la zona de rechazo y por lo tanto se acepta la hipótesis de investigación.

El segundo instrumento aplicado fue la entrevista la misma que fue aplicada a tres docentes del área de francés de la carrera plurilingüe de la Universidad Central del Ecuador. Se ejecutaron cinco preguntas relacionadas a las dimensiones de la variable independiente y se realizó un análisis cualitativo de las cinco respuestas obtenidas.

Las respuestas del personal docente no estuvieron alejadas en lo que respecta a los indicadores de las fases del aprendizaje por tareas. La teoría establece tres fases las mismas que se ejecutan con la tarea previa (pre task) en donde se introduce el tema de estudio; la tarea, que es la ejecución de la misma a través de la comunicación espontánea con el conocimiento previo y; la fase después de la tarea (post tarea), en la que se realiza tareas comunicativas utilizando el conocimiento previo y nuevo a través de recursos auténticos del idioma.

Se resalta también que para actividades de producción oral, los/las docentes entrevistados dan mayoritariamente prioridad al uso pragmático del idioma dentro de un contexto siendo necesariamente un requisito esencial la verificación del objetivo planteado al inicio de las fases de la tarea.

El uso de material auténtico por parte del personal docente encuestado, se evidencia más restringido a juego de roles o situaciones en donde la imaginación juega un papel muy importante 
para motivar la creatividad del estudiantado. La autenticidad de la tarea se enfoca a que se debe preparar a la persona para enfrentar situaciones que suceden en la vida real o en el diario vivir.

Los resultados que se pretender alcanzar al ejecutar una tarea, depende del objetivo específico planteado. El éxito de una tarea es conseguir que el estudiantado utilice el idioma de manera espontánea promoviendo cada vez más el aprendizaje del mismo. Los/las docentes manifestaron que el rol fundamental que tanto el/la profesor/a y el/la estudiante deben desempeñar son cruciales en el proceso de enseñanza aprendizaje. El/la docente, debe identificar las necesidades de los/las aprendices, debe convertirse en un proveedor, monitor y que solo interviene cuando es necesario permitiendo al estudiantado convertirse en sujetos autónomos en donde el aprendizaje surja a través de la interacción e intercambio de experiencias para la ejecución de la tarea.

La información obtenida tanto en la aplicación del pre test y post test, así como también, en la aplicación de la entrevista aportó significativamente para sustentar los objetivos planteados en esta investigación.

\section{Conclusiones.}

La presente investigación surge con la hipótesis sobre la influencia del método aprendizaje basado en tareas en la producción oral del idioma francés nivel B1 en los/las estudiantes de quinto semestre de la carrera plurilingüe en la Universidad Central del Ecuador. El método se aplicó en el periodo 2017 - 2017 de acuerdo al calendario académico establecido en la institución. Como se indica en el plan de intervención, luego de obtener la 
autorización por parte de las autoridades de la carrera plurilingüe, se estableció como punto de partida la aplicación del pre test tanto para el grupo experimental como de control. Se aplicó en el grupo experimental el método de aprendizaje basado en tareas en la producción oral durante ocho semanas mientras que con el grupo de control se continuó con el método sugerido por libro Alter Ego+3. Se aplicó el post test al grupo experimental y control, instrumento que evidenció notablemente la influencia en el grupo experimental del aprendizaje basado en tareas en la producción oral (Grupo experimental=21,03/25 Grupo control=16,81/25) al realizar el cálculo de la prueba paramétrica $\mathrm{Z}$ se determinó que la hipótesis nula está en la zona de rechazo y por lo tanto se aceptó la hipótesis alterna de investigación.

El personal docente de la carrera plurilingüe está consciente que para ejecutar una tarea hay que sondear el conocimiento previo, a través de una lluvia de ideas, para asegurarse que la misma haya sido entendida para su ejecución. Sin embargo, esta constituye solo la primera fase llamada la tarea previa (pre task) en donde el/la docente introduce el tema y constata que sus estudiantes estén muy claros/as en las instrucciones. Luego se debe continuar con la tarea, utilizando el conocimiento previo para ejecutar la misma priorizando tanto la motivación como la comunicación espontánea y finalmente la fase después de la tarea (post task) en donde se sistematiza el conocimiento previo con el conocimiento adquirido para la realización de tareas comunicativas orales a través de documentos auténticos.

En lo que respecta a los factores que influyen en el aprendizaje basado en tareas para la producción oral se identifica el ámbito de la tarea, los/las docentes de la carrera plurilingüe dan más importancia a la pragmática del idioma ya que el uso del idioma se lo hace en contexto y su producción es integral y secuencial a la adquisición de un conocimiento nuevo o la utilización de un 
conocimiento previo para lograr los objetivos planteados del idioma meta. Otro factor identificado, es la perspectiva de la tarea, ningún docente manifestó explícitamente realizar un plan de trabajo (workplan) para estructurar las actividades y direccionarlas hacia el objetivo planteado. Las instrucciones son fundamentales ya que se especifica el propósito de la tarea y lo que el estudiantado debe hacer para alcanzar el resultado. El factor de la autenticidad de la tarea también juega un papel importante dentro del método de aprendizaje basado en tareas, los/as docentes del área de francés toman en cuenta este factor para motivar la creatividad de sus estudiantes a fin de que puedan comunicarse de manera natural pero la autenticidad de la tarea además de propiciar la imaginación de los/las aprendices debe prepararlos/as para situaciones que enfrentarán en su diario vivir y fuera del aula de clase.

Los componentes del aprendizaje basado en tareas identificados en la presente investigación son las metas a las que aspira que el estudiantado logre alcanzar de manera natural. La meta de una tarea tiene que ser establecida en términos de contenido. Como lo manifiesta el personal docente del área de francés, no es primordial que el estudiantado logre metas exitosas sino que usen el idioma como una herramienta que lo motive a seguir aprendiendo. Sin embargo, al tener un sílabo que completar, los/las docentes se ven muchas veces obligados/as a poner más énfasis en los resultados de aprendizajes establecidos en silabo y evidentemente, estas metas tienen que verse reflejadas con parámetros cuantitativos a través de calificaciones. Otro componente identificado es el rol que cumplen el/la docente y el/la estudiante. Los/as docentes de la carrera plurilingüe manifiestan que el rol que deben desempeñar debe ser en función a las necesidades del estudiantado. Monitorean el desempeño de sus estudiantes en las tareas asignadas siendo el centro de atención ya que son un ente autónomo en la ejecución de la tarea asignada. 
Los resultados obtenidos demuestran que el nivel de producción oral de los/las estudiantes de quinto semestre de la carrera plurilingüe del periodo 2017 - 2017 es B1 según el Marco Común Europeo de Referencia para las Lenguas con un promedio para el grupo de control de 16,01/25 y para el grupo experimental 21,03/25. Es evidente que los dos resultados demuestran que el estudiantado posee el nivel B1. Sin embargo, al aplicar el método de aprendizaje basado en tareas al grupo experimental, los resultados fueron aún más satisfactorios. Por consiguiente, el aprendizaje basado en tareas influye en el nivel de producción oral del francés en los/las estudiantes de quinto semestre.

\section{Bibliografía.}

Cháux Grajales, V. (2002). Mercadeo sin economía... ¿existe? Revista Colombiana de Marketing, 3(5), 8-13.

Gorbaneff, Y. (2001). Teoria de la agencia y sus aplicaciones en el mercadeo. INNOVAR, revisla de eiencias administrativas y sociales, 18(1), 37-46.

Gorbaneff, Y. (2003). TeorÌa del Agente-Principaly el mercadeo. REVISTA Universidad EAF, $129(1), 75-86$.

Herrera, L., Medina, A., Naranjo, G., \& Proaño, J. (2002). Tutoría de la Investigación. Quito: AFEFCE.

Lavayen, L. (2007). Formulación e Informe de Proyectos de Investigación. Quito: Publicación UCE.

Sabino, C. (1994). Como hacer una tésis y elaborar todo tipo de escritos. Caracas: Panapo.

Sampieri Hernández, R., Collado Fernández, C., \& Lucio Baptista, P. (2003). Metodología de la Investigación. México: McGraw-Hill Interamericana.

Tamayo y Tamayo, M. (2003). El Proceso de la Investigación Científica. Mexico: Limusa.

Vélez Zapata, C., \& Hardy Fioravanti, R. (2009). La etnografía como un acercamiento interdiscipLinario en el mercadeo: un nuevo intento. Cuadernos de Administración, 22(38), 101-119. 